\title{
Les deux épisodes caniculaires de l'été 2019 à Grenoble : constat et perspective pour une gestion des extrêmes thermiques futurs
}

\author{
Sandra Rome ${ }^{1 *}$, Sylvain Bigot ${ }^{1}$, Xavier Foissard ${ }^{2}$, Malika Madelin ${ }^{3}$, Sarah Duche ${ }^{4}$ et Anne-Cécile Fouvet ${ }^{5}$ \\ ${ }^{1}$ Université Grenoble Alpes, CNRS, IRD, Grenoble INP, UMR 5001 IGE, Grenoble, France \\ ${ }^{2}$ Université Grenoble Alpes, IUGA, Grenoble, France ; Université Rennes 2, TerraClima/LETG Rennes, UMR 6554 CNRS, Rennes, France \\ ${ }^{3}$ Université de Paris, UMR 8586 PRODIG, Paris, France \\ ${ }^{4}$ Université Grenoble Alpes, UMR 5194 PACTE, Grenoble, France \\ ${ }^{5}$ Direction de l'Environnement et du Cadre de Vie, Ville de Grenoble, France
}

Résumé - Cette étude détaille la spécificité des deux vagues de chaleurs qui se sont produites à l'échelle de la ville de Grenoble (Isère, France) en juin et juillet 2019. Les données thermiques traitées regroupent 7 stations, en particulier certaines encore jamais analysées dans une perspective d'analyse climatologique. Les analyses portent aussi sur les valeurs thermiques quotidiennes projetées à l'échelle de l'aire urbaine en fonction du scénario RCP 8.5 sur la période 2006-2100. Les résultats confirment que la ville possède une grande variabilité spatiale lors d'un événement chaud extrême, que le réseau de mesure actuel n'arrive pas encore à discriminer suffisamment, sans compter les contrastes verticaux qui existent également. Les vagues de chaleur de 2019 sont a priori des analogues potentiels des conditions attendues d'ici 2050 (alors que l'aléa exceptionnel de 2003 serait sûrement représentatif de la normale de fin du siècle). Ces retours d'expérience et éléments scientifiques quantifiés sur l'aléa caniculaire aident la Ville de Grenoble, très impliquée dans l'étude du risque climatique, à améliorer et anticiper ses programmations et son urbanisme tactique pour s'adapter au changement climatique, en se construisant son propre diagnostic typique des caractéristiques microclimatiques urbaines.

Mots-clés : canicule / été 2019 / Grenoble / réseau in situ / projections climatiques / stratégies urbaines.

\begin{abstract}
The two heat waves of summer 2019 in Grenoble: observation and perspective for a management of future thermal extremes. This study details the specificity of the two heat waves that occurred in the city of Grenoble (Isère, France) in June and July 2019. The processed temperature data includes 7 stations, in particular some that have never before been analysed from a climatological analysis perspective. The analyses also cover the daily temperature values projected at the scale of the urban area according to the RCP 8.5 scenario over the period 2006-2100. The results confirm that the city possesses a high degree of spatial variability during an extreme hot event, which the current measurement network is not yet able to discriminate sufficiently, not to mention the vertical contrasts that also exist. The heat waves of 2019 are a priori potential analogues of the conditions expected between now and 2050 (whereas the exceptional heat wave of 2003 would surely be representative of the normal at the end of the century). These feedbacks and quantified scientific elements on the heat wave hazard help the City of Grenoble, which is very involved in the study of climate risk, to improve and anticipate its programming and tactical urban planning to adapt to climate change, by building its own typical diagnosis of urban microclimatic characteristics.
\end{abstract}

Keywords: heat wave / summer 2019 / Grenoble / in situ network / climate projections / urban strategies.

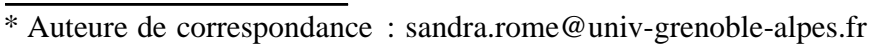




\section{Introduction}

Les villes ont un potentiel unique pour s'adapter rapidement aux risques liés aux extrêmes thermiques, à condition d'entreprendre des analyses sur ce risque encore mal défini à l'échelle locale et en élaborant des plans de réduction et de gestion spécifiques aux différents cas urbains (Singh et al., 2019). Mais l'absence d'informations cohérentes sur les villes reste un obstacle majeur dans la science du climat urbain pour informer et développer des planifications d'atténuation et d'adaptation à cette échelle (Baklanov et al., 2020). Le fait est que l'état des lieux climatologique, pour ensuite l'intégrer à des stratégies d'adaptation au changement climatique, peut encore être considéré comme un thème très récent pour les planificateurs urbains (Musco et al., 2016). Le saut d'échelle entre des constats et diagnostics génériques provenant de résultats et d'organismes nationaux ou internationaux n'est pas toujours utile et suffisant (Bigot et al., 2017). Les défis de l'urbanisme stratégique (qui cible les actions et évolutions sur les infrastructures physiques) ou tactique (qui s'intéresse plus à la vision sociale et à la place de l'habitant) ne pourront être correctement relevés que si l'identification de l'aléa thermique extrême est clairement définie et connue, ce qui n'est pas le cas pour la majorité des villes, surtout celles ne bénéficiant pas d'un réseau et/ou d'un système de veille météo-climatique.

Seule une connaissance précise des variations climatiques locales passées, en cours ou projetées, peut permettre de développer des composantes adaptées aux cas urbains. De nombreux pays européens ont commencé des études de leurs territoires urbains à la suite des vagues de chaleur subies en 2003 et 2006 (Chao et al., 2010). A l'échelle de la métropole de Lyon par exemple, Alonso et Renard (2020) montrent des vulnérabilités physiologiques des populations aux vagues de chaleur plus élevées au centre-ville, tandis que celles socio-économiques s'observent en périphérie de l'agglomération. L'échelle climatique locale apparaît ainsi essentielle pour construire la ville et son orientation en fonction de l'évaluation $\mathrm{du}$ futur climat urbain, les stratégies de développement à intégrer correspondant à des échelles idéalement situées entre $100 \mathrm{~m}$ et $1 \mathrm{~km}$ (Foissard et al., 2019).

Actuellement il existe trois grandes méthodes portées par la modélisation statistique et/ou numérique pour étudier et évaluer le climat urbain ainsi que ses interactions avec les différentes composantes physiques ou anthropogéniques du changement climatique: 1) par le biais des analogues climatiques, 2) avec la descente d'échelle statistico-dynamique provenant des projections numériques du climat, 3) en ayant recours au couplage à haute résolution spatiale entre les modèles urbains et les modèles atmosphériques (Daniel, 2017). Cet auteur indique qu'une quatrième méthode reste essentielle, celle s'appuyant sur l'étude des événements extrêmes $a$ priori représentatifs du climat futur, sachant que tous les résultats actuels issus de la modélisation numérique indiquent des vagues de chaleur plus longues et intenses en France à moyen et surtout à long terme (Ouzeau et al., 2016 ; Suarez-Gutierrez et al., 2020).

A l'échelle européenne, l'année 2019 est la plus chaude depuis l'origine des relevés, suivie de près par 2014, 2015 et 2018 (Copernicus, 2020). L'été 2019 correspond au quatrième plus chaud depuis 1979, avec des anomalies thermiques dépassant 3 à $4^{\circ} \mathrm{C}$ dans certaines régions, notamment à cause des deux vagues de chaleur très intenses enregistrées en juin et juillet. En France métropolitaine, elles dépassent les seuils d'alerte entre le 24 juin et le 7 juillet, puis entre le 21 et le 27 juillet, et pour la première fois, respectivement 4 et 20 départements (soit 7 et $35 \%$ de la population française) sont placés en vigilance rouge par Météo France (BSP, 2019). La surmortalité dans les départements placés en vigilance rouge (dont ceux de la Région Auvergne-Rhône-Alpes) est environ $50 \%$ plus élevée que la moyenne des départements touchés (572 décès en excès), avec plus de 12000 hospitalisations d'après l'indicateur national iCanicule suivi dans le cadre du 'Plan National Canicule' (PNC).

Ce caractère estival exceptionnel observé et ressenti en 2019 à l'échelle de la ville de Grenoble donne alors typiquement l'opportunité d'un premier retour d'expérience pour d'une part, décrypter la 
temporalité climatique de cette séquence extrême chaude estivale, et d'autre part, analyser comment la ville, en s'appropriant les études et résultats climatologiques, peut déjà mettre en place, améliorer ou anticiper des mesures (adaptatives, réglementaires, techniques...) face à ces aléas thermiques (Rome et al., 2020). L'objectif est également de pouvoir juger, d'après les connaissances actuelles, si ce type d'événement correspond à ceux envisageables à court et moyen termes déterminés grâce aux projections numériques du climat, élément clé pour améliorer les systèmes de veille et d'alerte, à l'instar de ceux existant en Amérique du Nord, dès 1995 à Philadelphie (Etats-Unis) où le $1^{\text {er }}$ système d'alerte canicule a été mis en place, ou encore les «Avertissements de chaleur extrême » émis par l'Institut national de santé publique du Québec (INSPQ).

\section{Site d'étude et données thermiques utilisées}

\subsection{Le réseau météorologique sélectionné}

Le bassin de vie grenoblois $\left(1^{\mathrm{er}}\right.$ pôle urbain alpin français comprenant 196 communes et plus de 687000 habitants début 2020) est représentatif des grandes zones urbaines possédant un réseau météoclimatique peu dense et sans chronique longue représentative lui permettant de détailler et reconstituer un diagnostic spatio-temporel précis, par exemple pour l'étude de son îlot de chaleur urbain ou de ses spécificités topoclimatiques diurnes et intra-saisonnières (Bigot et al., 2017). En conséquence, cette étude cherche dans un premier temps à maximiser les points de mesures possibles en 2019 en s'appuyant sur le réseau de Météo France mais aussi sur d'autres nouvelles stations météorologiques institutionnelles de l'aire grenobloise. Les séries de six stations couvrant l'ensemble de l'agglomération de Grenoble (figure 1) ont ainsi pu être collectées, ainsi qu'une septième située à plusieurs kilomètres de la ville et utilisée comme pseudo-référence pour le climat grenoblois au sein du réseau national (tableau 1) :

- trois stations du réseau Météo France dont les données sont accessibles via la Publithèque (publitheque.meteo.fr/) : 1) Saint-Etienne-de-Saint-
Geoirs (Aéroport), existante depuis 1940 mais située à $48 \mathrm{~km}$ au nord-ouest de la ville de Grenoble dans un environnement rural de surcroît à $384 \mathrm{~m}$ d'altitude (contre $210 \mathrm{~m}$ pour la commune grenobloise) ; elle ne peut donc absolument pas être utilisée comme référentiel urbain (malgré sa qualité métrologique de niveau 0 ), même si son nom reste toujours "Grenoble-Saint-Geoirs" dans le référentiel de Météo France et que ses relevés sont régulièrement utilisés abusivement pour décrire la variabilité météo-climatique du site urbain, puisqu'étant la seule longue série interannuelle disponible ; 2) Le Versoud (dite Grenoble-LVD), station synoptique (de qualité 1) installée depuis juin 1999 sur l'aérodrome éponyme, et servant de station de référence urbaine sur Grenoble jusqu'à l'automne 2019 ; 3) Grenoble-CEA-Radôme intégrée en mars 2019 au réseau national (de qualité 1), sachant que cette station était préalablement opérationnelle et installée par l'Institut Max von Laue - Paul Langevin (ILL) du CEA. L'apport conjoncturel de cette station désormais disponible et située sur le site urbain du Polygone Scientifique, dans la cluse de l'Isère ventilée, est essentiel pour une définition améliorée du climat local. En outre, elle permet également une étude météo-climatique très fine de la stratification des températures atmosphériques puisqu'elle mesure cette variable à 2, 10, 20, 60 et 100 mètres de hauteur.

- trois stations provenant du réseau de mesure Atmo Auvergne-Rhône-Alpes, l'observatoire agréé par le Ministère de la Transition écologique et solidaire, pour la surveillance et l'information sur la qualité de 1'air : 1) Les Frênes (station urbaine), 2) Le Rondeau (périurbaine), 3) Saint-Martin-d'Hères (urbaine de fond). Les séries ne sont pas collectées dans des conditions métrologiques standard de l'OMM puisque les parcs de mesures sont dédiés en priorité à la mesure de la qualité de l'air. Les fiches stationnelles sont consultables ici : atmoauvergnerhonealpes.fr/donnees/acces-par-station/20055

- une station installée par l'Institut des Géosciences de l'Environnement (IGE) depuis août 2016 et située sur le toit d'un bâtiment de recherche (bâtiment OSUG-B) sur le campus universitaire de Saint-Martin-d'Hères. Les métadonnées et données sont consultables en ligne ici : ige-grenoble.fr/-mesures-en-temps-reel-. 


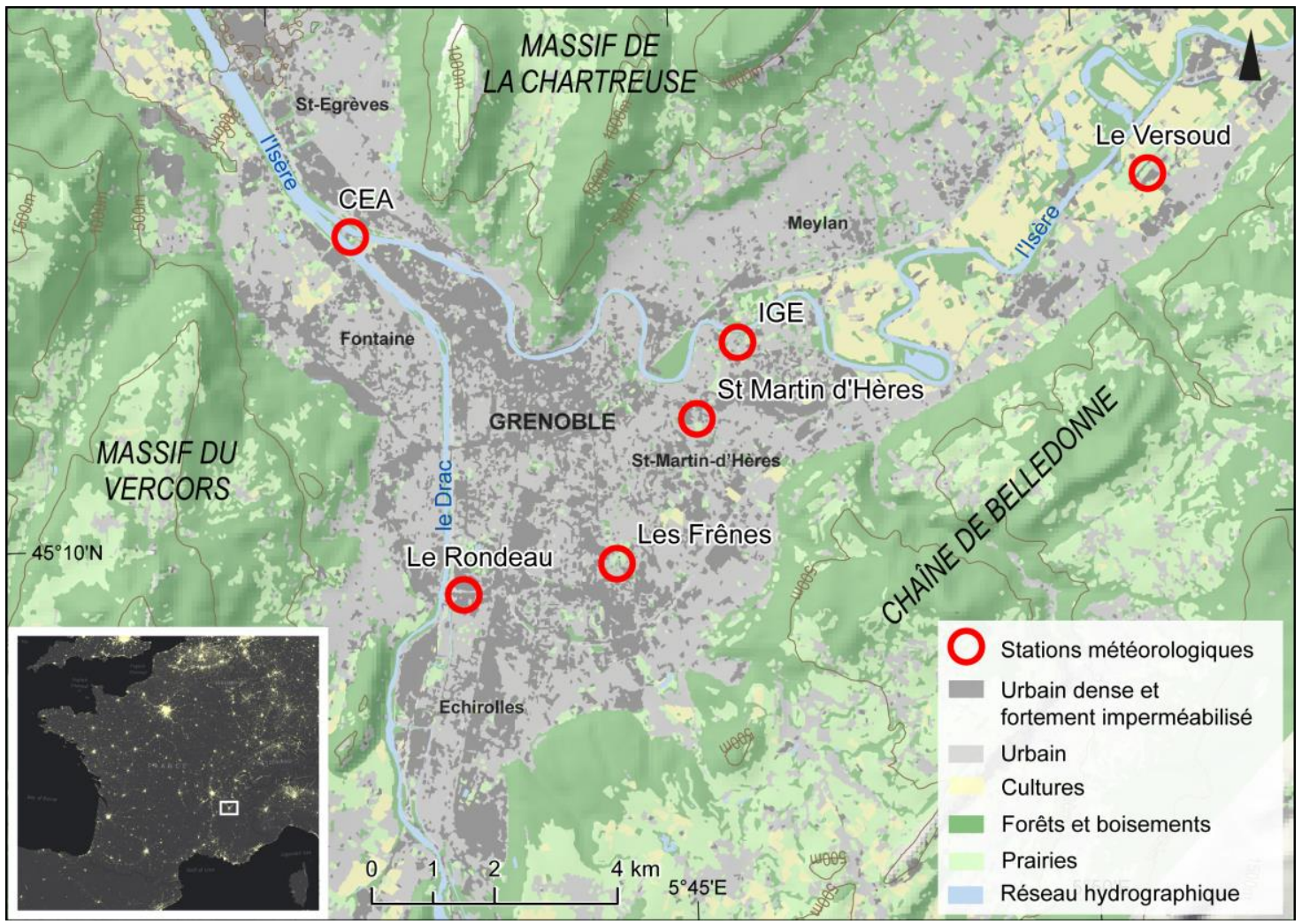

Figure 1. Localisation des stations météorologiques étudiées à Grenoble (Saint-Geoirs non visible). Sources des données cartographiques : IGN BD ALTI (2018) et DDT de l'Isère (2015). Location of the meteorological stations studied in Grenoble (Saint-Geoirs not visible). Sources of the data: IGN BD ALTI (2018) and DDT de l'Isère (2015).

Tableau 1. Caractéristiques des séries thermiques analysées sur la période du 1er juin au 31 août 2019 à l'échelle de Grenoble. Characteristics of the temperature time series analysed over the period from June 1st to August 31 st 2019 at the Grenoble scale.

\begin{tabular}{|lcccc|}
\hline Nom de la station (nom d'usage et/ou particularité) & $\begin{array}{c}\text { Altitude } \\
(\mathbf{m})\end{array}$ & $\begin{array}{c}\text { Variable } \\
\left({ }^{\circ} \mathbf{C}\right)\end{array}$ & $\begin{array}{c}\text { Pas de } \\
\text { temps }\end{array}$ & Source \\
\hline Saint-Etienne-de-Saint-Geoirs (Aéroport Saint-Geoirs) & 384 & $\mathrm{Tn}, \mathrm{Tx}$ & quotidien & Météo France \\
\hline CEA-Radôme (Grenoble CEA)* & 213 & $\mathrm{~T}$ & 6 à $12 \mathrm{~min}$ & $\mathrm{CEA}$ \\
\hline Le Versoud (Aérodrome) & 220 & $\mathrm{Tx}, \mathrm{Tx}$ & quotidien & Météo France \\
\hline Grenoble Les Frênes (Les Frênes) & 214 & $\mathrm{~T}$ & $15 \mathrm{~min}$ & Atmo AURA \\
\hline Le Rondeau (Rocade sud) & 219 & $\mathrm{~T}$ & $15 \mathrm{~min}$ & Atmos AURA \\
\hline Saint-Martin-d'Hères & 219 & $\mathrm{~T}$ & $15 \mathrm{~min}$ & Atmo AURA \\
\hline IGE (+ hauteur du toit du bâtiment OSUG-B) & $213+15$ & $\mathrm{~T}$ & $10 \mathrm{~min}$ & IGE \\
\hline
\end{tabular}

* La station Grenoble-CEA fournit des données de température à 2, 10, 20, 60 et 100 mètres de hauteur. The GrenobleCEA station provides temperature data at 2, 10,20,60 and 100 meters.

\subsection{Les réanalyses atmosphériques et sorties de modèles}

Afin de contextualiser la variabilité climatique interannuelle régionale et celle sur le plan national ou international, les séries thermiques mensuelles provenant des réanalyses NCEP-NCAR sur la période 1948-2020 sont analysées (Kalnay et al.,
1996), collectées librement auprès du Physical Science Laboratory (psl.noaa.gov/data/). Elles servent également au calcul des cinq principales normales climatiques trentenaires ensuite intercomparées (1951-1980, 1961-1990, 1971-2000, 1981-2010 et 1991-2019).

Les séries sur le climat futur correspondent aux 
chroniques des températures minimales (Tn) et maximales (Tx) corrigées quotidiennes pour l'été (JJA) 2019 du point de grille SAFRAN défini pour Grenoble (figure 2), calculé à partir du modèle CNRM-ALADIN-Climat pour la période 2006-

Figure 2. Aire de $5 \mathrm{~km}$ autour de Grenoble correspondant au point de grille SAFRAN (point 7394) ayant servi à calculer les données Tn et Tx, d'altitude moyenne 284 mètres, pour le climat futur (2006$2100)$; d'après un extrait du portail Drias. Area of $5 \mathrm{~km}$ around Grenoble corresponding to the SAFRAN grid point (point 7394) used to calculate the Tn and Tx data for future climate (2006-2100), with an average altitude of 284 meters; from Drias portal.
2100 en fonction du scénario RCP 8.5, le plus représentatif des situations climatiques extrêmes attendues. L'ensemble de ces données est obtenu librement à partir du portail Drias de Météo France (www.drias-climat.fr/).

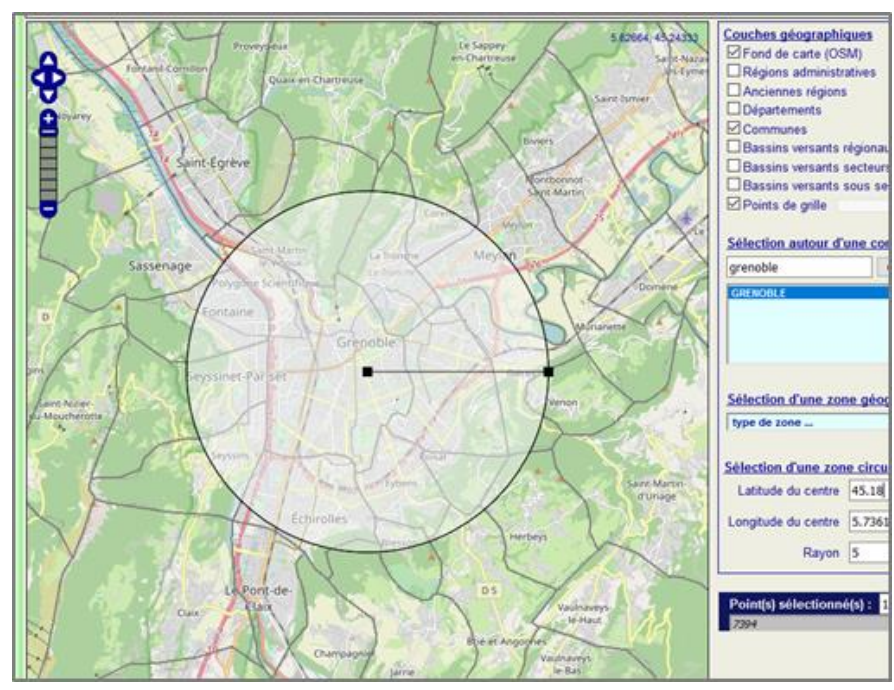

\subsection{Les deux vagues de chaleur de l'été 2019 à Grenoble}

Les définitions sur la notion de vague de chaleur (et de canicule souvent utilisée en France) sont nombreuses et pas toujours homogénéisées à ce jour, car multicritères et reposant sur différentes formulations et objectifs analytiques (cf. par exemple Bador, 2016 ; Soubeyroux et al., 2016 ; Rome et al., 2020 pour plus de détails conceptuels). La méthode élaborée par Météo France (2020) pour définir une vague de chaleur s'appuie sur les données de l'indicateur thermique national (une moyenne de 30 stations régulièrement réparties sur le territoire) disponibles depuis 1947. La durée de l'événement est ensuite calculée à partir d'une valeur seuil, caractérisant le début et la fin de l'épisode.

Pour l'Isère, la station de référence utilisée par Météo France est Saint-Geoirs (située en milieu rural), avec respectivement les seuils Tn et Tx de 19 et $34^{\circ} \mathrm{C}$. Le terme de « canicule » est essentiel pour les collectivités car il permet de définir la mise en place de mesures spécifiques à partir du niveau 3 de l'alerte canicule (PNC, 2017). nationale, la canicule enregistrée un peu avant en
juin 2019 arrive au troisième rang par son intensité, mais toujours sur une durée de 6 jours. 


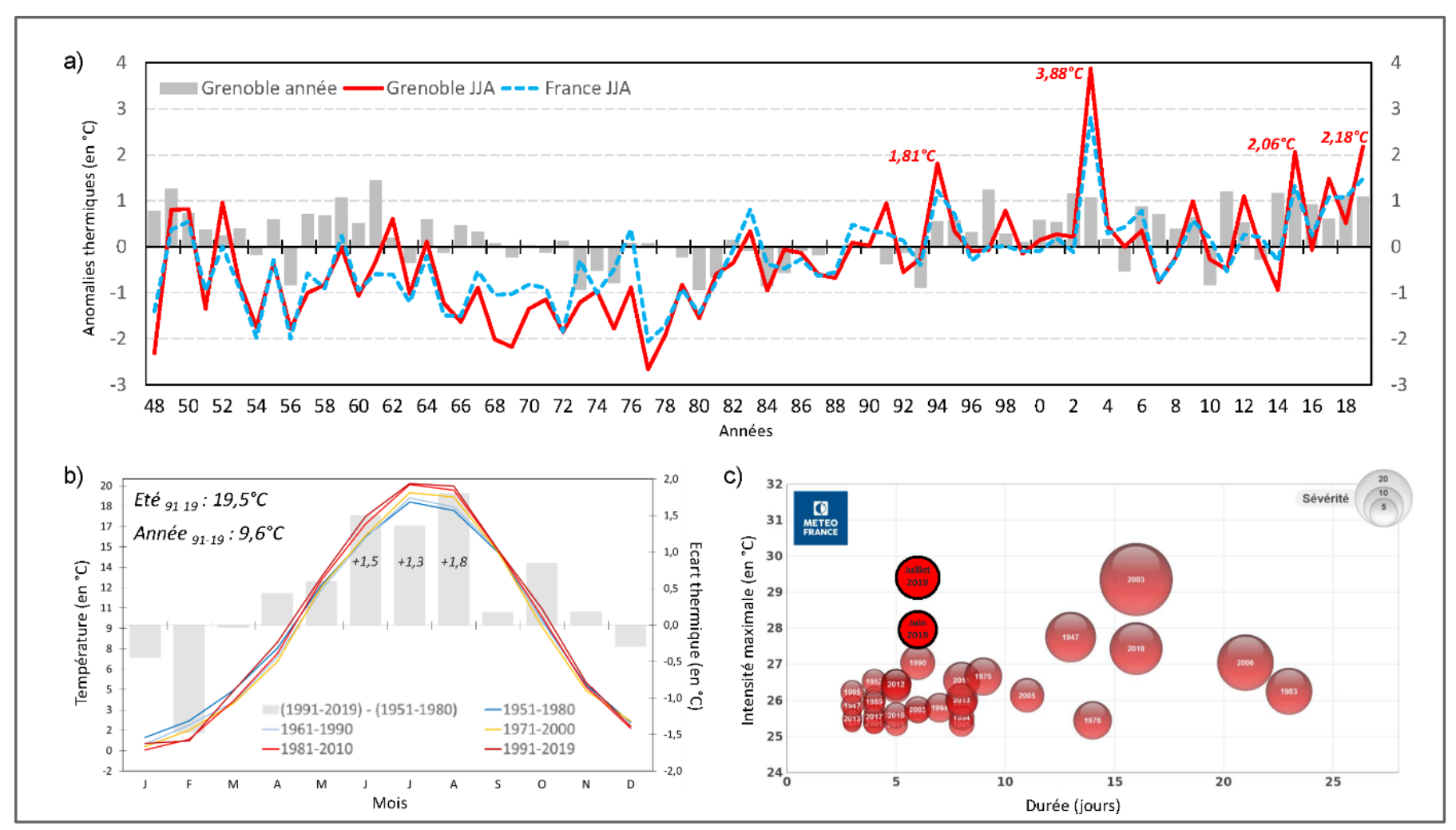

Figure 3. a) Anomalies des températures moyennes (à 2 m) entre 1948 et 2019 à l'échelle de l'aire grenobloise (pour l'année moyenne et juin-juillet-août) ou de la France métropolitaine (pour juin-juillet-août) calculées d'après les réanalyses NCEP-NCAR et selon la normale climatique 1981-2010; b) Régime annuel moyen des températures à $2 \mathrm{~m}$ pour l'aire grenobloise calculé pour cinq normales climatiques trentenaires échelonnées sur la période 1951-2019 (le différentiel des normales 1991-2019 moins 1951-1980 est indiqué en histogramme) ; c) Les 41 épisodes de vague de chaleur observés en France (1947-2019) selon leur intensité-durée-sévérité, dont les épisodes de juin et juillet 2019 (adapté d'après Météo France). (a) Temperature anomalies (at 2m) between 1948 and 2019 for the Grenoble area (for the average year and June-July-August average) or for Metropolitan France (for June-July-August average) calculated from NCEP-NCAR reanalyses and according to the 1981-2010 climate normal; b) Mean annual temperature regime at $2 m$ for the Grenoble area calculated for five three-decade climate normal over the 1951-2019 period (the differential of the 1991-2019 minus 1951-1980 normal is shown in histogram); c) The 41 heat wave episodes observed in France (1947-2019) according to their intensity-duration-severity, including the June and July 2019 episodes (adapted from Météo France).

Comme partout en France, les températures de l'été 2019 sont particulièrement élevées, avec des anomalies diurnes dépassant $+3,5^{\circ} \mathrm{C}$ en région Auvergne-Rhône-Alpes (d'après le bulletin saisonnier de Météo France). A l'échelle régionale, à partir des huit stations de référence du réseau national, Météo France détermine pour 2019 une anomalie thermique de $+2,4^{\circ} \mathrm{C}$ en juin (soit le $3^{\text {ème }}$ rang des mois les plus chauds depuis 1970, après juin 2003 avec $+5,6^{\circ} \mathrm{C}$ et juin 2017 avec $+3,4^{\circ} \mathrm{C}$ ), et $\mathrm{de}+2,4^{\circ} \mathrm{C}$ en juillet ( $4^{\text {ème }}$ rang des mois de juillet les plus chauds depuis 1969, après juillet 2015 et 2006 avec $+3,4^{\circ} \mathrm{C}$, et juillet 1983 avec $+3,2^{\circ} \mathrm{C}$ ). Deux vagues de chaleur majeures sont enregistrées à l'échelle de la région grenobloise : la première du
24 juin au 2 juillet, et la seconde du 21 au 27 juillet (figure 4). Presque toutes les stations étudiées enregistrent des valeurs anormalement élevées durant ces périodes, mais c'est dans le sud de la ville (stations Le Rondeau et surtout Les Frênes) que les températures sont les plus élevées, avec des pics supérieurs à $40^{\circ} \mathrm{C}$, et même un maximum absolu de $43^{\circ} \mathrm{C}$ à $15 \mathrm{~h} 30$ le 23 juillet. Les nuits sont aussi anormalement chaudes, avec par exemple 44 nuits «tropicales » (i.e. nombre de nuits où $\mathrm{Tn} \geq 20^{\circ} \mathrm{C}$ ) enregistrées à la station Le Rondeau (figure 5), contre 4 enregistrées au Versoud, station périurbaine autrefois utilisée comme référence par Météo France pour définir les conditions et la variabilité du climat urbain grenoblois (Rome et al. 
2020). Un troisième épisode chaud de type caniculaire, mais de moindre ampleur, est également enregistré localement les 4-5-6 août (figure 4).

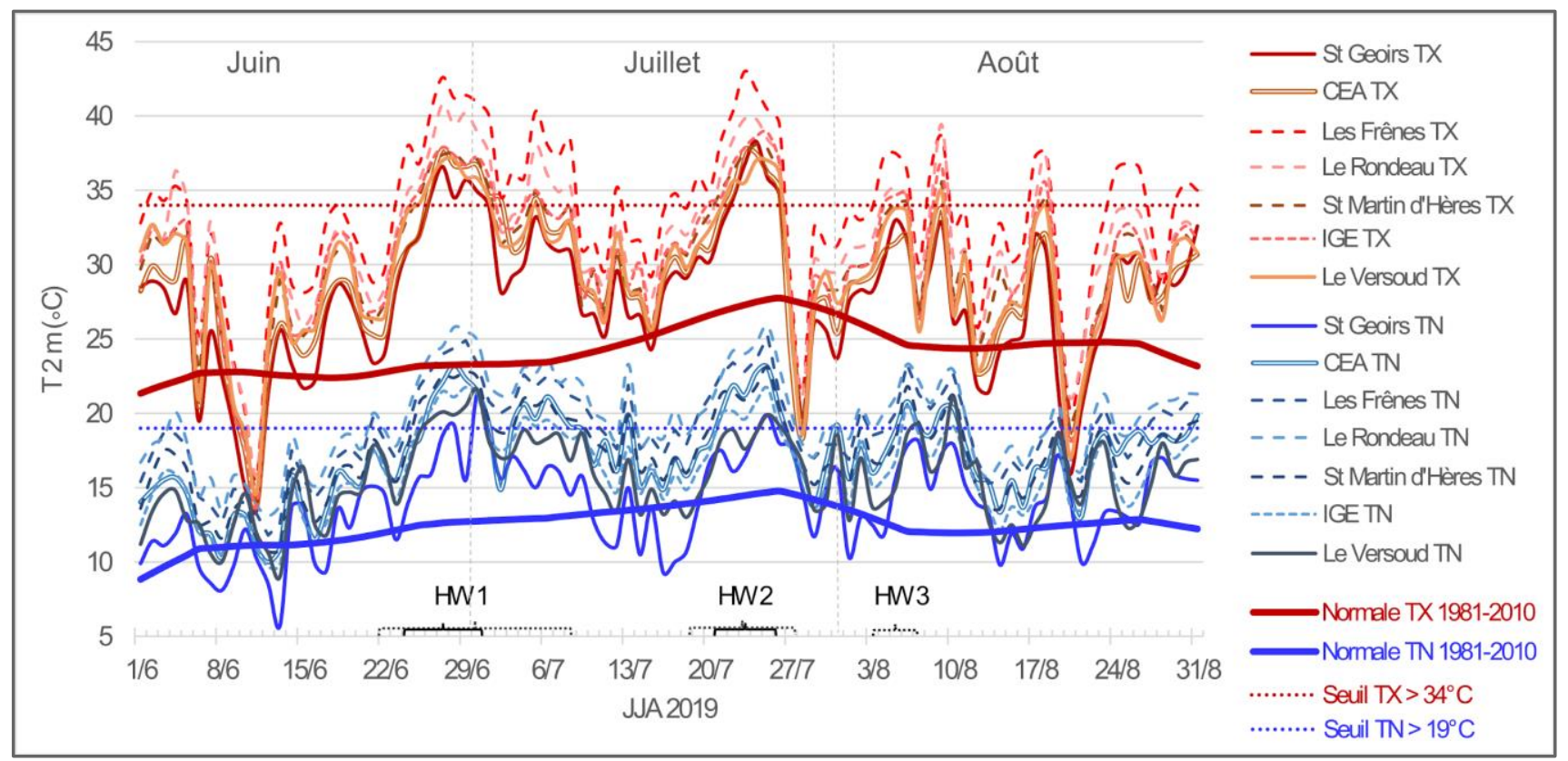

Figure 4. Fluctuations des températures estivales (JJA 2019) journalières dans l'aire grenobloise. HW (pour vague de chaleur) 1 à 3 signalent la présence de 3 canicules ; les parenthèses en trait plein indiquent les canicules enregistrées dans toutes les stations, les parenthèses en pointillé celles enregistrées dans quelques stations. Les normales 1981 2010 de Saint-Geoirs sont représentées en trait épais. Les seuils biométéorologiques $T N>19^{\circ} \mathrm{C}$ et $\mathrm{TX}>34^{\circ} \mathrm{C}$ pour l'Isère sont indiqués. Source des données : Météo France, CEA, IGE et Atmo AURA. Daily summer temperature fluctuations (JJA 2019) in Grenoble area. HW (Heat Wave) 1 to 3 indicate the presence of the 3 heat waves; the brackets indicate the heat waves recorded in all stations, the dashed brackets indicate those recorded in some stations. The 1981-2010 normals for Saint-Geoirs are shown in thick lines. Biometeorological thresholds $T N>19{ }^{\circ} \mathrm{C}$ and $T X>34^{\circ} \mathrm{C}$ for Isère are indicated. Data source: Météo France, CEA, IGE and Atmo AURA.

La deuxième vague de chaleur apporte des records durant 3 à 4 jours sur toute l'Europe Occidentale, avec par exemple pour la première fois en Belgique et aux Pays-Bas des températures supérieures à $40^{\circ} \mathrm{C}$ (Vautard et al., 2019). Quoique plus court au Royaume-Uni (seulement 2 jours), l'aléa chaud a dépassé la précédente valeur record journalière historique d'août 2003. A partir d'une approche en modélisation numérique, Vautard et al. (2019) montrent que contrairement à d'autres vagues de chaleur déjà enregistrées en Europe de l'Ouest, cette vague extrême de juillet 2019 est un événement rare pour le climat actuel en France métropolitaine, les températures observées sur 3 jours possédant une période de retour se situant entre 50 et 150 ans.

Ainsi, malgré l'évènement chaud record historique très homogène à l'échelle synoptique européenne, lorsqu'on se focalise à l'échelle du site urbain grenoblois, il demeure toujours de forts contrastes thermiques dépendant des conditions de surface (habitat, quartiers, ventilation, végétalisation), avec des écarts moyens ou des maximum maximorum de plusieurs degrés Celcius (figure 5), comme cela a déjà été observé lors de la canicule de juillet 2015 (Bigot et al., 2016). Alors qu'en moyenne, la station rurale de Saint-Geoirs enregistre bien la plus faible valeur estivale, elle subit la même valeur extrême $\left(38,3^{\circ} \mathrm{C}\right)$ que les stations périurbaines ou urbaines. Légèrement plus chaude en moyenne, la station péri-urbaine Le Versoud enregistre les plus faibles records de températures $\left(37,1^{\circ} \mathrm{C}\right)$. Ce diagnostic confirme que les réponses et mesures d'adaptation urbaines doivent être envisagées par quartier, car la vulnérabilité thermique des habitants est conditionnée par ce zonage thermique complexe, qui ne peut pas être considéré comme un ensemble 
homogène répondant au pic des températures caniculaires imposé par les conditions météorologiques synoptiques, ni à un simple découpage rural/périurbain/urbain lors des maxima diurnes. Cette amplitude des températures, même lors d'une phase chaude extrême, indique aussi que les stations de référence utilisées par Météo France, celle de Le Versoud depuis 1999 et celle de CEARadôme depuis fin 2019, ne sont pas représentatives des conditions thermiques les plus extrêmes subies par la partie urbaine et centrale de Grenoble.

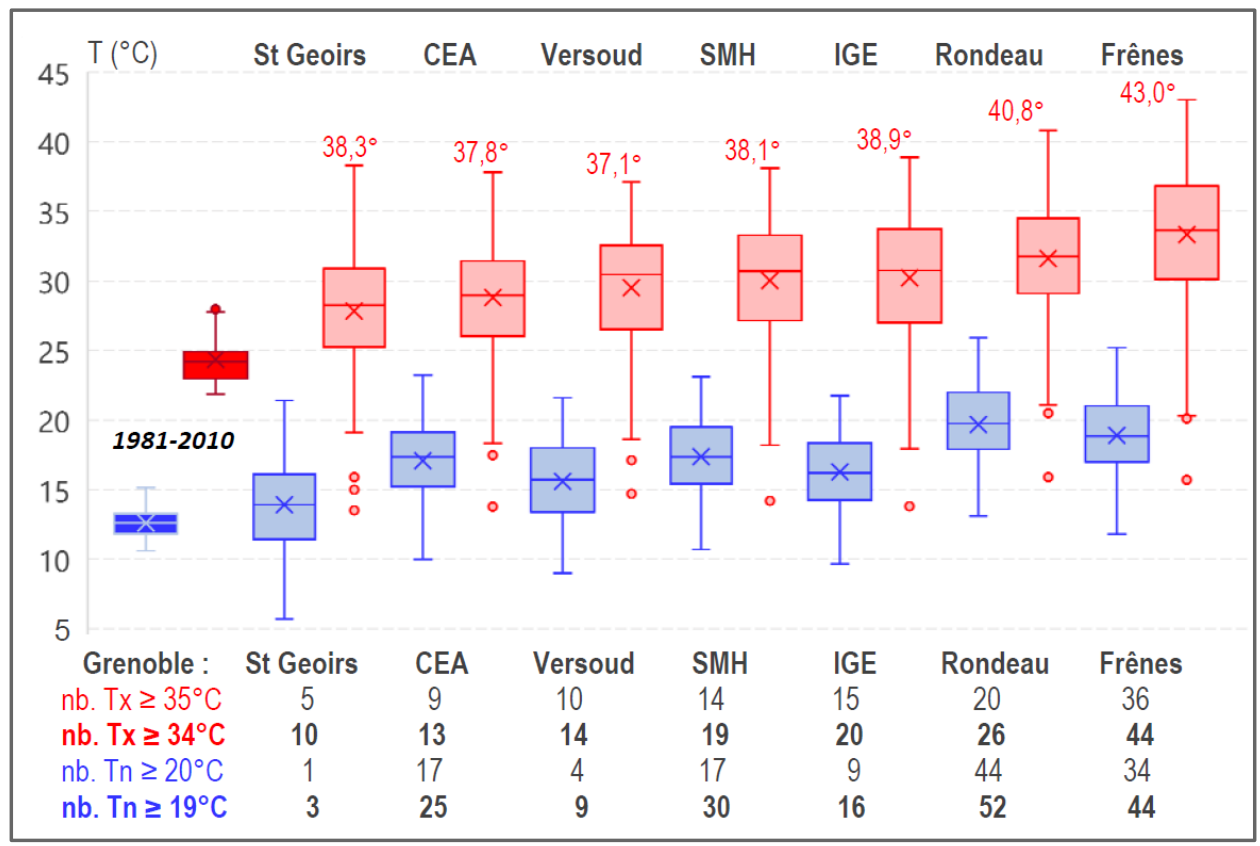

Figure 5. Variations thermiques journalières des Tx (en rouge) et des Tn (en bleu) en JJA 2019 pour 7 stations du site grenoblois classées par ordre croissant de la valeur du maximum maximorum des Tx (indiquée en rouge) ; le nombre de jours très chauds $\left(T x \geq 35^{\circ} \mathrm{C}\right)$, de nuits tropicales $\left(T n \geq 20^{\circ} \mathrm{C}\right)$ et des seuils biométéorologiques de l'Isère $\left(T x \geq 34^{\circ} \mathrm{C}\right.$ et $\mathrm{Tn} \geq 19^{\circ} \mathrm{C}$ en italique) est indiqué en bas (calculé d'après des données de Météo France, Atmo AURA, CEA et IGE). Daily thermal variations of Tx (in red) and Tn (in blue) in JJA 2019 for 7 stations of the Grenoble area classified in ascending order of the value of the maximum maximorum of $T x$ (indicated in red); the number of very hot days $\left(T x \geq 35^{\circ} \mathrm{C}\right)$, tropical nights $\left(T n \geq 20^{\circ} \mathrm{C}\right)$ and biometeorological thresholds of Isère $\left(T x \geq 34^{\circ} \mathrm{C}\right.$ and $T n \geq 19^{\circ} \mathrm{C}$ in italics) is indicated at the bottom (calculated from data from Météo France, Atmo AURA, CEA and IGE).

\subsection{Précisions stationnelles lors de la première vague de chaleur fin juin 2019}

Lors de l'analyse des processus météorologiques urbains, il est essentiel de représenter l'échange de la quantité de mouvement entre la surface et l'atmosphère sus-jacente (Oke, 1982 ; Theeuwes et $a l ., 2019)$. Les progrès dans la simulation du confort thermique, de la qualité de l'air et des mécanismes météo-climatiques dépendent alors d'observations précises et de la modélisation des processus au sein de la sous-couche de rugosité (roughness sublayer), cruciale car à l'interface entre la surface et l'atmosphère fortement influencée par les activités (Barlow et al., 2014 ; Daniel, 2017). Cette couche dans laquelle l'écoulement et les flux turbulents sont perturbés par les obstacles peut être de deux à cinq fois la hauteur moyenne de la canopée urbaine, sachant qu'à l'échelle de la commune de Grenoble, la hauteur moyenne du bâti est de 14,3 m, et de 18,5 $\mathrm{m}$ dans le centre-ville ancien. La mise à disposition des données de la station CEA-Radôme depuis fin 2019 offre désormais la possibilité de détailler cette stratification thermique des basses couches en zone urbaine grenobloise, particulièrement lors d'épisodes extrêmes comme celui de l'été 2019, alors que toutes les précédentes études fondées sur l'observation ou la modélisation développent plutôt une approche à l'échelle supérieure de la vallée par manque de données intra-urbaines (Largeron, 2010). 
Les profils mensuels moyens verticaux calculés en 2019 pour le site CEA-Radôme indiquent que les températures sont relativement cohérentes dans la sous-couche située entre 2 et $100 \mathrm{~m}$ de hauteur, avec une légère décroissance souvent inférieure à $1^{\circ} \mathrm{C}$ (figure 6). Si l'on analyse le cas des mois estivaux, on observe que les températures les plus élevées sont celles mesurées à $10 \mathrm{~m}$ (et pas à $2 \mathrm{~m}$, comme cela est généralement le cas dans les stations de mesure), avec un différentiel moyen de 0,2 à $0,4^{\circ} \mathrm{C}$ entre les deux hauteurs, et respectivement des valeurs moyennes de $22,5^{\circ} \mathrm{C}$ en juin, $25,2^{\circ} \mathrm{C}$ en juillet et $23,1^{\circ} \mathrm{C}$ en août. Cela provient a priori du microclimat du parc instrumental situé à proximité de deux cours d'eau (Drac et Isère) et avec une hauteur de bâti moyenne plus faible (4,2 m) qu'en espace typiquement urbain, pouvant influencer la stratification des températures. Cela suggère donc qu'au-delà des forts contrastes thermiques horizontaux constatés auparavant à l'échelle de l'aire urbaine grenobloise (effet du bâti sur les vents locaux), il y a aussi potentiellement un différentiel moyen significatif entre le niveau du sol $(2 \mathrm{~m})$ et celui plus élevé vers $10 \mathrm{~m}$ correspondant plus ou moins à la canopée urbaine.

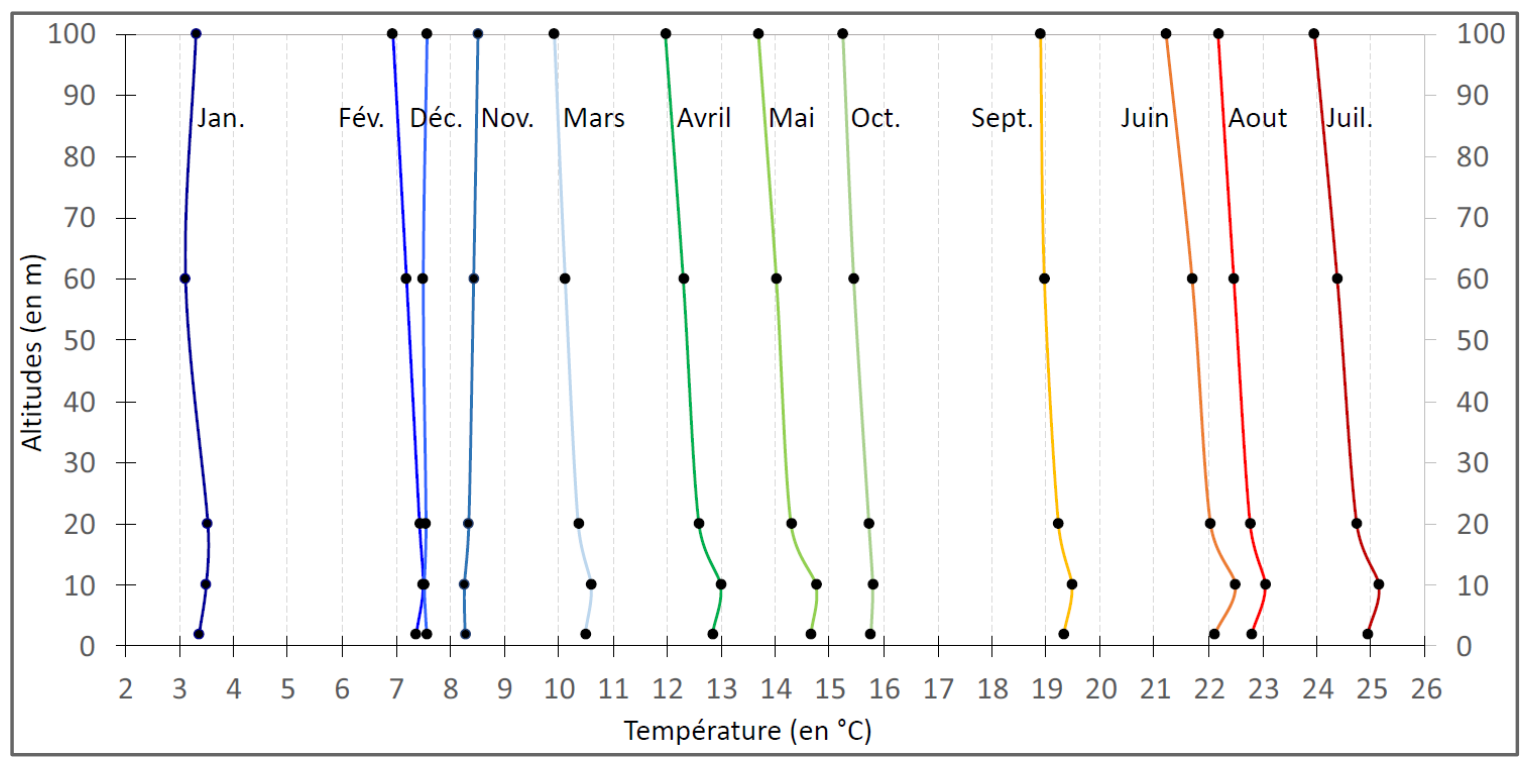

Figure 6. Profils verticaux des températures moyennes mensuelles (en ${ }^{\circ} \mathrm{C}$ ) en 2019 pour 5 niveaux d'altitude $(2,10$, 20,60 et $100 \mathrm{~m}$ ) à la station météorologique Grenoble-CEA-Radôme. Vertical profiles of average monthly temperatures (in ${ }^{\circ} \mathrm{C}$ ) in 2019 for 5 altitude levels (2, 10, 20, 60 and 100m) at the Grenoble-CEA-Radôme meteorological station.

L'analyse plus précise des variations du cycle nycthéméral lors de la première vague de chaleur débutant le 25 juin et se terminant le 2 juillet montre que lors $\mathrm{du}$ pic caniculaire (le 27/06), les températures diurnes au-dessus du seuil d'alerte $\left(34^{\circ} \mathrm{C}\right)$ durent de $11 \mathrm{~h} 30$ jusqu'à $19 \mathrm{~h} 30$, alors que le jour précédent, elles ne duraient que de 15 à $18 \mathrm{~h}$ (figure 7a). Et en dehors des deux jours marquant le début et la fin de vague, les températures nocturnes ne descendent jamais en-dessous du seuil d'alerte de $19^{\circ} \mathrm{C}$. Cela révèle qu'au sein même de la vague de chaleur extrême, en dehors des contrastes territoriaux, la temporalité des contraintes thermiques diurnes varie également rapidement, ce qui demande un suivi quotidien précis et réactualisé de la part des collectivités pour adapter les activités et mesures réglementaires.

Là encore, l'apport de nouvelles stations urbaines facilite ce suivi spatio-temporel et cette mise à jour régulière en conditions extrêmes. Si on se fonde sur la station de référence périurbaine de Le Versoud (qui servait aux calculs des procédures d'alerte caniculaire, par exemple pour planifier l'adaptation des phases de travail, l'accueil du public ...), on voit que lors du pic extrême du 27 juin, les températures au-dessus du seuil d'alerte sont légèrement plus faibles que celles du site CEARadôme, elles-mêmes proches des valeurs enregistrées par la station Saint-Martin d'Hères (figure $7 b$ ). 


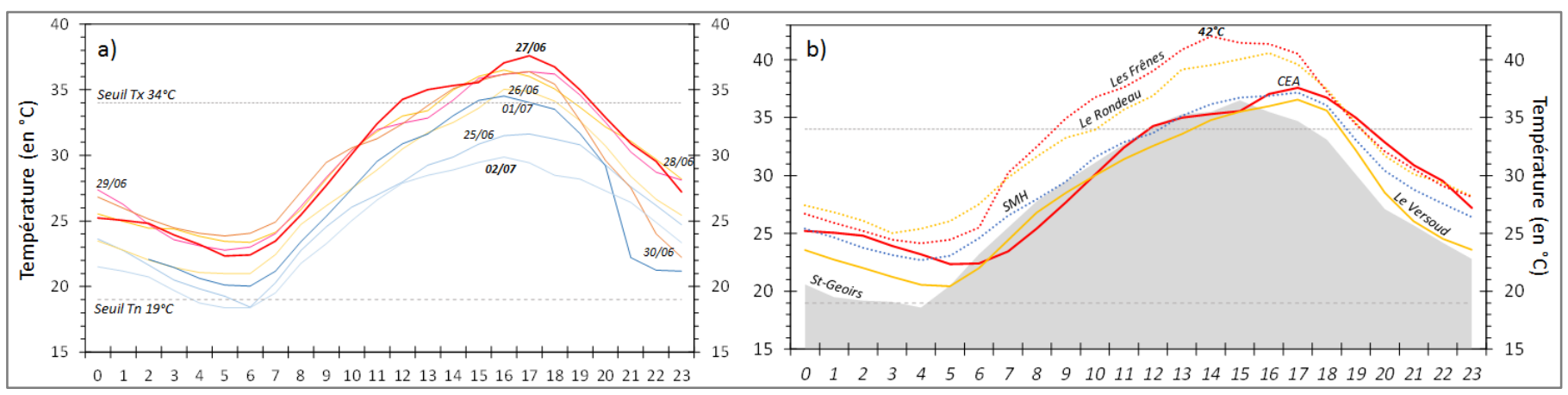

Figure 7. Cycle thermique nycthéméral (en valeur horaire moyenne) : a) pour la station météorologique Grenoble-CEARadôme durant l'épisode caniculaire observé sur la période du 25 juin au 2 juillet 2019 ; b) pour une comparaison de 6 stations le jour du pic caniculaire le 27 juin 2019 (la station rurale de Saint-Geoirs est représentée en surface grisée ; les stations CEA-Radôme et Le Versoud issues du réseau Météo France sont en courbe continue ; les stations issues du réseau Atmo AURA sont en courbe pointillée) ; les seuils d'alerte biométéorologiques de 19 et $34^{\circ} \mathrm{C}$ sont indiqués par des traits horizontaux. Nycthemeral thermal cycle (hourly average value): a) for the Grenoble-CEA-Radôme meteorological station during the heatwave episode observed over the period from 25 June to 2 July 2019; b) for a comparison of 6 stations on the heatwave peak on 27 June 2019 (the rural station of Saint-Geoirs is shown in shaded area ; the CEA-Radôme and Le Versoud stations from the Météo France network are shown in a continuous curve; the stations from the Atmo AURA network are shown in a dotted curve); the biometeorological alert thresholds of 19 and $34^{\circ} \mathrm{C}$ are indicated by horizontal lines.

En revanche, les deux stations caractéristiques des espaces urbains plus denses et centraux (Le Rondeau et Les Frênes) enregistrent des températures diurnes, mais également nocturnes, largement supérieures aux précédentes, avec des différences pouvant dépasser $7^{\circ} \mathrm{C}$ en milieu de journée, alors que les stations sont seulement distantes de $6,5 \mathrm{~km}$ (et même si on tient compte des différences métrologiques évoquées en $\$ 1.1$.). Ces éléments descriptifs confirment que les procédures, algorithmes et décisions des autorités et tutelles sanitaires lors des phases caniculaires doivent donc plutôt chercher à fonder leur expertise sur des séries météo-climatiques typiques des conditions vécues, et pas sur les chroniques d'une station moins représentative de cette variabilité haute fréquence en phase caniculaire.

\subsection{L'été 2019, un avant-goût des étés futurs à Grenoble ?}

Le retour d'expérience à partir des chroniques in situ permet de qualifier le détail et la temporalité des phases caniculaires, et vient conforter les approches phénoménologiques pratiquées et souvent très bien maîtrisées par les collectivités, dans un objectif $a$ posteriori d'améliorer et mieux caler les systèmes d'alerte, de prévention et d'adaptation. Mais le diagnostic doit également être complété par une perspective des conditions futures attendues en s'appuyant sur les projections et expertises des modèles numériques du climat, ce qui est à ce jour plus technique et ardu à réaliser par les collectivités. Pourtant, l'OMM a adopté en 2018 une approche urbaine transversale comme l'une de ses priorités dans un objectif de durabilité et de résilience, suggérant le concept de services urbains intégrés (ou Urban Services Survey) pour favoriser les études fondées sur l'observation et les scénarios futurs, rassemblant hydro-météorologie et climatologie urbaine, services environnementaux, systèmes urbains d'alerte précoce multirisques, informations intégrées sur les gaz à effet de serre et services climatiques (Baklanov et al., 2020).

L'épisode chaud exceptionnel enregistré à Grenoble en 2019 donne ainsi un exemple pertinent de comparaison possible par rapport aux projections thermiques futures du GIEC issues du scénario RCP 8.5 (i.e. le plus extrême en forçage radiatif, donc en réchauffement potentiel), pour déterminer dans quelle mesure et fréquence la ville de Grenoble devra faire face à des vagues de chaleur de la même intensité voire d'ampleur supérieure. Le choix est fait ici de ne retenir que les résultats provenant du modèle ALADIN, alors qu'une approche ensembliste multi-modèle serait évidemment plus robuste. Mais d'une part, après inter-comparaison 
(résultats non montrés), les projections issues du modèle ALADIN corroborent très largement celles provenant des autres modèles disponibles à partir du portail Drias de Météo France; d'autre part, car l'objectif est à terme de pouvoir intégrer cette expertise dans une orientation de service climatique pour la collectivité, l'enjeu n'est pas de mener une étude exhaustive et d'inter comparaison de la qualité des modèles mais bien d'en proposer un pertinent avec des données régulièrement mises à jour et accessibles, comme c'est le cas avec ALADIN.

L'analyse des valeurs maximales des températures diurnes ( $\mathrm{Tx}$ ) ou nocturnes (Tn) projetées à l'échelle du bassin grenoblois sur le $21^{\text {ème }}$ siècle d'après le scénario RCP 8.5 indique plusieurs éléments significatifs (figure 8): $i$ ) une hausse jusqu'au milieu du siècle des Tn estivales qui dépassent régulièrement le seuil de $19^{\circ} \mathrm{C}$, et des Tx particulièrement plus élevées en août; ii) une augmentation très nette du réchauffement dans la seconde moitié du siècle, pour les températures nocturnes de tous les mois d'été, mais aussi très largement des Tx en juillet et août (tableau 3) ; iii) également après 2050, une augmentation très significative de la durée des vagues de chaleur $\left(>34^{\circ} \mathrm{C}\right)$, passant par exemple en moyenne en août de 1 jour sur la période 2006-2050 à 12,2 jours sur 2051-2100.

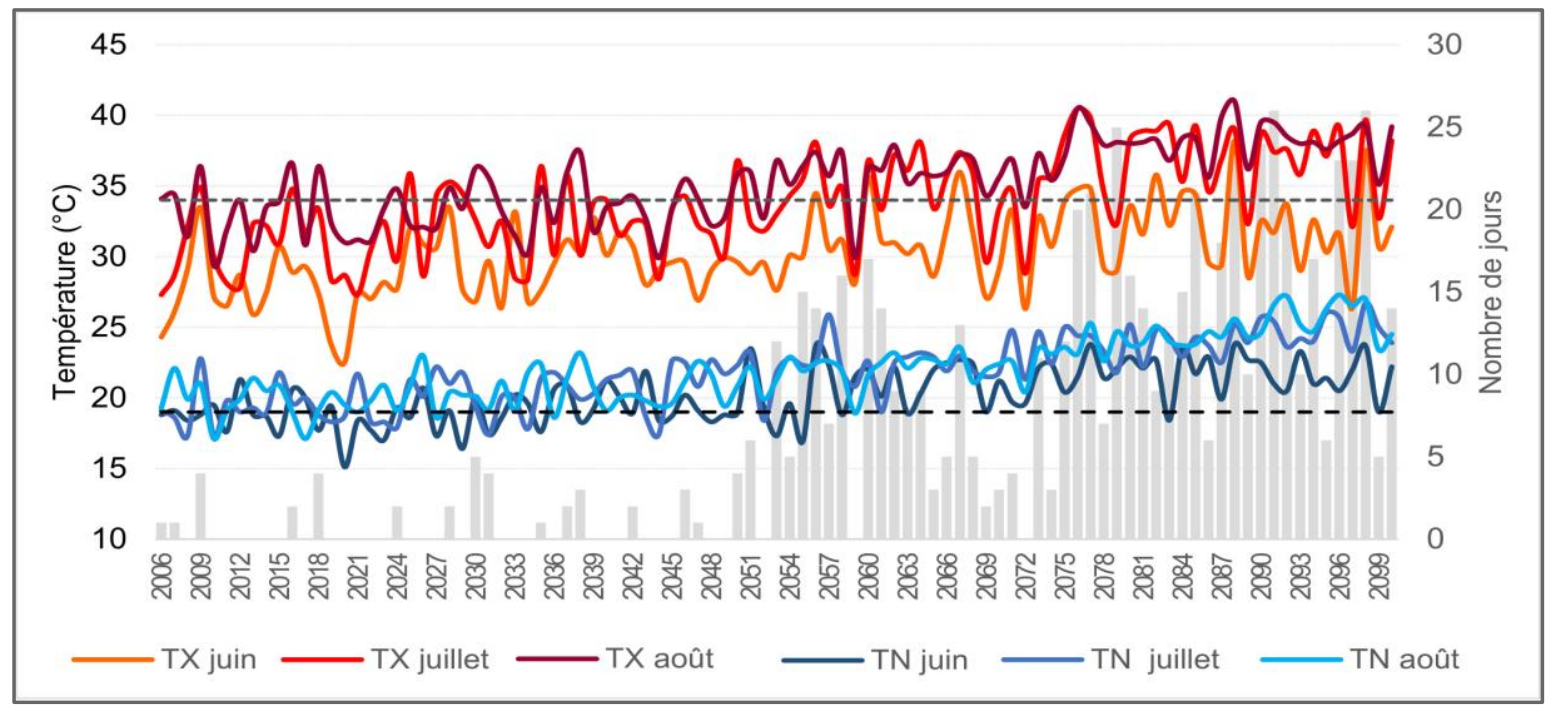

Figure 8. Évolution des valeurs maximales des températures (à $2 \mathrm{~m}$ ) pour les Tn et les Tx des mois de juin, juillet et août sur la période future 2006-2100 pour le point de grille Grenoble-Safran issu du modèle ALADIN, et selon le scénario RCP 8.5 (l'histogramme représente le nombre de jours en août susceptibles de dépasser le seuil biométéorologique $\mathrm{TX}>34^{\circ} \mathrm{C}$; les seuils $\mathrm{TN}>19^{\circ} \mathrm{C}$ et $\mathrm{TX}>34^{\circ} \mathrm{C}$ sont indiqués par les traits pointillés horizontaux ; source des données : Drias-Climat). Evolution of maximum temperature values (at $2 \mathrm{~m}$ ) for Tn and Tx for June, July and August over the future period 2006-2100 for the Grenoble-Safran grid point from the ALADIN model, and according to the RCP 8.5 scenario (the histogram represents the number of days in August likely to exceed the biometeorological threshold $T X>34^{\circ} \mathrm{C}$; the thresholds for $T N>19^{\circ} \mathrm{C}$ and $T X>34^{\circ} \mathrm{C}$ are indicated by horizontal dashed lines; data source: DriasClimat).

Tableau 3. Tendance linéaire de la température minimale (Tn) et maximale (Tx) de l'air (à $2 \mathrm{~m}$ ) pour le point de grille Grenoble-Safran (modèle ALADIN) sur les deux sous-périodes futures 2006-2050 et 2051-2100, selon le scénario RCP 8.5 (calculée d'après les données obtenues sur le portail Drias). Linear trend of air temperature (at $2 \mathrm{~m}$ ) for the GrenobleSafran grid point (ALADIN model) over the two future sub-periods 2006-2050 and 2051-2100, according to the RCP 8.5 scenario (calculated from data obtained on the Drias portal).

\begin{tabular}{|c|c|c|c|c|}
\hline & \multicolumn{2}{|c|}{ Tendance des $\operatorname{Tn}\left(\mathrm{en}^{\circ} \mathrm{C}\right)$} & \multicolumn{2}{|c|}{ Tendance des $\operatorname{Tx}\left(\mathrm{en}^{\circ} \mathrm{C}\right)$} \\
\hline & $2006-2050$ & $2051-2100$ & $2006-2050$ & $2051-2100$ \\
\hline Juin & $+1,96$ & $+1,40$ & $+2,55$ & $+1,29$ \\
\hline Juillet & $+1,88$ & $+3,91$ & $+1,50$ & $+4,66$ \\
\hline Août & $+0,99$ & $+4,43$ & $+0,57$ & $+3,71$ \\
\hline
\end{tabular}


Avec le scénario de réchauffement modéré RCP 4.5, et à partir de nouvelles données modélisées (Max Planck Institute Grand Ensemble), SuarezGutierrez et al. (2020) projettent qu'à la fin du $21^{\text {ème }}$ siècle en Europe (avec évidemment des disparités régionales), un mois d'été moyen sera comparable au mois le plus chaud observé au cours des vagues de chaleur de la période 1981-2010, les évènements estivaux extrêmes futurs pouvant atteindre des valeurs deux fois plus importantes que celles enregistrées vers 2010. L'analyse fréquentielle comparant les deux vagues de chaleur récentes très intenses 2003 et 2019 (que l'on peut théoriquement considérer comme des analogues pertinents des conditions futures), et les températures moyennes estivales, apporte plusieurs précisions à l'échelle grenobloise (figure 9) :

- les 2 évènements observés ne sont pas vraiment similaires en durée-intensité, notamment pour les Tx, rappelant bien qu'il n'y a pas une 'canicule type' modélisable à l'échelle locale ;

- pour les Tn futures, les périodes chaudes nocturnes excéderont très largement celles observées en 2003 et 2019, la fin du siècle enregistrant par exemple potentiellement plus de 35 nuits d'été supérieures à $22^{\circ} \mathrm{C}$;

- pour les températures diurnes, on peut considérer que la vague de chaleur de 2019 a des similitudes avec les conditions estivales projetées à moyen terme, alors que celle de 2003 est plutôt à mettre en correspondance avec les températures des étés de fin du siècle, et en insistant bien sur le fait que le nombre de jours chauds futurs va considérablement augmenter (la classe modale pour 2021-2050 étant de 20 jours en JJA avec $29^{\circ} \mathrm{C}$, contre 23 jours avec $33^{\circ} \mathrm{C}$ en $2071-2100$, alors qu'elle n'est que de $25^{\circ} \mathrm{C}$ pour la normale 1991-2019).

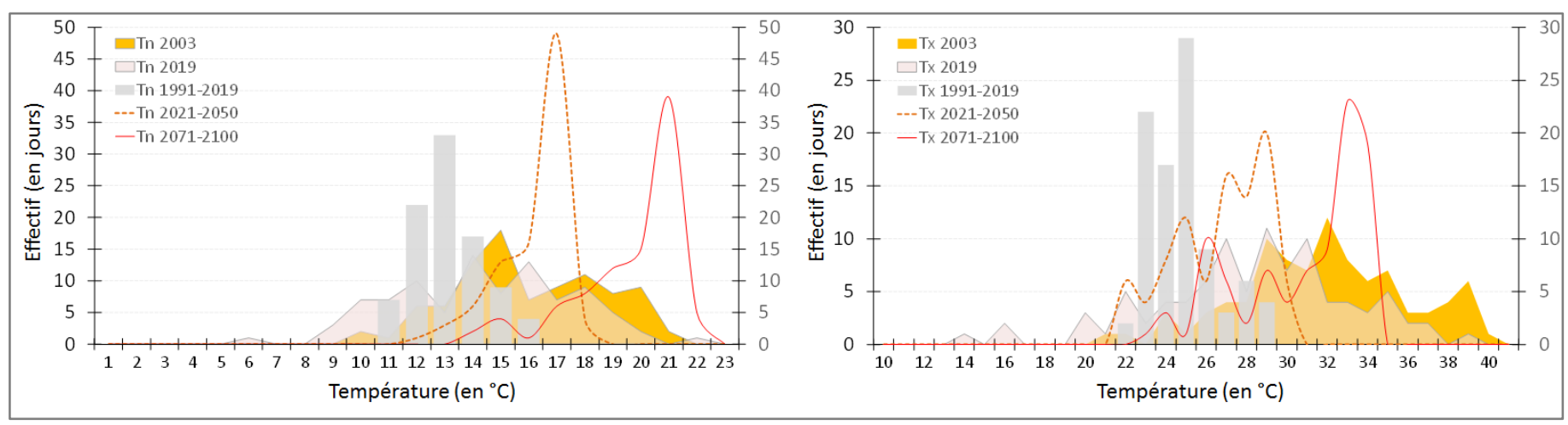

Figure 9. Variations fréquentielles des températures (à $2 \mathrm{~m}$ ) minimales (Tn à gauche) et maximales (Tx à droite) en été (JJA) pour les années caniculaires 2003 et 2019 ainsi que pour la normale estivale 1991-2019 (calculées à partir de la station de Saint-Geoirs), et comparaison avec les fréquences des étés futurs moyens 2021-2050 et 2071-2100 (calculées à partir des données provenant du modèle ALADIN pour le scénario RCP 8.5 et le point de grille sur Grenoble). Frequency variations of minimum (Tn on the left) and maximum (Tx on the right) summer (JJA) temperatures (at $2 m$ ) for the 2003 and 2019 heatwaves and for the climate normal 1991-2019 (from the Saint-Geoirs station), and comparison with the frequencies of future average summers 2021-2050 and 2071-2100 (from the ALADIN model for the RCP 8.5 scenario and the grid point over Grenoble).

Ces éléments confirment donc localement qu'à l'échelle saisonnière, l'été extrême de 2003 est représentatif d'un été climatologique à l'horizon de la fin du $21^{\text {ème }}$ siècle, les évolutions des records chauds en JJA possédant un gradient méridional en Europe, avec les plus fortes augmentations sur le pourtour méditerranéen (cf. Bador, 2016 pour une revue exhaustive). Cette auteure détermine par exemple à partir de différentes projections numériques, une augmentation des maximorum thermiques estivaux de $7,7^{\circ} \mathrm{C}$ dans le Sud de la France, ce que l'on retrouve à l'échelle de Grenoble avec une valeur proche de $8^{\circ} \mathrm{C}$. En partant également du scénario RCP 8.5, Ouzeau et al. (2016) recensent en moyenne entre 21 et 40 vagues de chaleur sur la période 2021-2050. Cela correspond à une augmentation d'environ 19 événements par rapport au nombre médian de la période 1976-2005. Pour la fin du siècle, le nombre de vagues de chaleur extrêmes augmenterait 
considérablement, avec un nombre médian de 87, soit 72 de plus qu'au cours de la période 1971-2005, leur durée variant entre 7 et 11 jours en 2021-2050, et plutôt entre 11 et 17 jours à la fin du siècle. Vautard et al. (2019) montrent que toutes les vagues de chaleur analysées jusqu'à présent en Europe au cours des dernières années $(2003,2010,2015,2017$, 2018 et donc 2019) sont plus probables et plus intenses en raison du changement climatique (même si le degré de probabilité dépend fortement de la définition retenue pour qualifier l'événement extrême). L'intensité de l'épisode de juillet 2019 est telle en Europe occidentale que les amplitudes observées auraient été extrêmement improbables sans le changement climatique. Cependant, certes liée de manière évidente à l'augmentation des températures moyennes mondiales, SuarezGutierrez et al. (2020) rappellent que l'augmentation des extrêmes chauds résulte aussi de rétroactions induites à différentes échelles, notamment celle régionale et inférieure, ce qui justifie pleinement les études urbaines.

\section{Discussion : intégrer ces retours d'expérience pour aider la Ville de Grenoble à faire face aux aléas thermiques}

\subsection{Rappels des différents 'Plans d'Actions' locaux sur le risque climatique}

Réputée pour les fortes amplitudes thermiques diurnes de fond de cuvette topographique, Grenoble s'est très tôt (dès 2005) munie d'un 'Plan Climat Local' (PCL), renforcé par un volet 'qualité de l'air' puis par un volet 'adaptation au changement climatique'; le Plan Air Energie Climat (PAEC 2016-19 puis 2019-25) de la ville s'articule avec le Plan Municipal de Santé de 2016 (grenoble.fr/472plan-municipal-de-sante.htm), et contribue à la démarche d'adaptation au changement climatique de la Métropole. Toutes ces actions ont permis de reformuler les finalités de la politique air-énergieclimat de la collectivité pour : 1) lutter contre le dérèglement climatique et la pollution atmosphérique ; 2) réduire la vulnérabilité du territoire et de ses habitants ; 3) agir avec les habitants et les acteurs du territoire pour un cadre de vie préservant santé et environnement.
En cas de fortes chaleurs, d'autres actions complémentaires municipales sont déclenchées.

\subsection{Premier apport : définir et fournir des indicateurs locaux de qualité}

L'analyse de l'épisode extrême de 2019 a révélé qu'il y a encore trop d'indicateurs et de définitions techniques inadaptées pour une gestion communale spécifique, car provenant d'analyses menées dans un cadre national ou régional. La définition spatiotemporelle des IBM (Indicateurs Bio Météorologiques) utilisés dans le cadre du Plan National Canicule (PNC, 2017) pourra donc sûrement encore être raffinée. Les expertises sont souvent 'importées', et même dans certains cas, trop générales et s'appuyant sur des sources parfois non définies. L'appui d'une analyse climatologique renforcée pour les services gestionnaires doit donc se consolider, par exemple grâce aux données du site CEA-Radôme qui permettra de mieux caler les algorithmes de climatologie urbaine fondés sur les produits de télédétection optique, avec une estimation des températures de surface souvent mal ou peu liée à celle des couches sus-jacentes.

\subsection{Second apport : développer une veille météo-climatique renforcée}

Nos résultats montrent que les stations utilisées pour l'instant comme source de calibration des études sur les aléas thermiques ne sont pas suffisamment représentatives des situations dans toute l'aire grenobloise. Pour améliorer la veille météo-climatique des extrêmes thermiques et la cartographie de l'îlot de chaleur, la Ville de Grenoble a donc décidé de réaliser une campagne de mesures durant l'été 2020 en s'appuyant sur l'expertise de climatologues et avec un réseau d'une trentaine de capteurs déportés (installés sur du mobilier urbain) ainsi que grâce à l'installation de trois stations météorologiques pérennes sur des sites représentatifs du centre-ville.

Dans une logique d'étude à l'échelle de l'aire métropolitaine, la ville voisine attenante d'Echirolles (36 840 habitants) a également entamé ce même protocole métrologique depuis l'été 2019, ce qui à terme pourrait rapidement renforcer les connaissances et les capacités locales pour 
modéliser les variations spatio-temporelles et raffiner les stratégies urbaines. C'est aussi une opportunité pour mieux mesurer et diagnostiquer les contraintes thermiques (réelles ou ressenties) au sein de la canopée urbaine lors de vagues de chaleur, enjeu essentiel de l'urbanisme tactique visé à Grenoble.

\subsection{Troisième apport : les leçons de la canicule 2019 pour préparer les étés futurs}

La prévention auprès des habitants commence par la mise en 'vigilance canicule' proposée par les services météorologiques; celle-ci guide la Préfecture de Police à activer le 'Plan Canicule départemental (Isère)'. Ainsi, Météo France a placé le département de l'Isère en vigilance orange canicule à compter du 24/06/2019 à 16 h puis à partir du 21 juillet. Le Préfet de l'Isère a donc activé le niveau 3 de l'alerte canicule (vigilance orange) à ces occasions, du 24 juin au 2 juillet et du 21 au 27 juillet (tableau 4). Les alertes canicules sont également souvent concomitantes au déclenchement, toujours par le Préfet, d'alertes pollution à l'ozone sur le bassin grenoblois.

Tableau 4. Journées de l'été 2019 durant lesquelles Météo France a placé le département Isère en 'vigilance canicule' (à gauche) et la Préfecture de l'Isère a activé des niveaux du 'plan canicule' (à droite) ; établies d'après les données du Centre Météorologique Interrégional de Lyon (CMIRCE) et la Préfecture de l'Isère. Days of summer 2019 during which Météo France placed the Isère department on 'heat wave vigilance' (left) and the Isère Prefecture activated levels of the 'heat wave plan' (right). Data from CMIRCE and Isère Prefecture of Police.

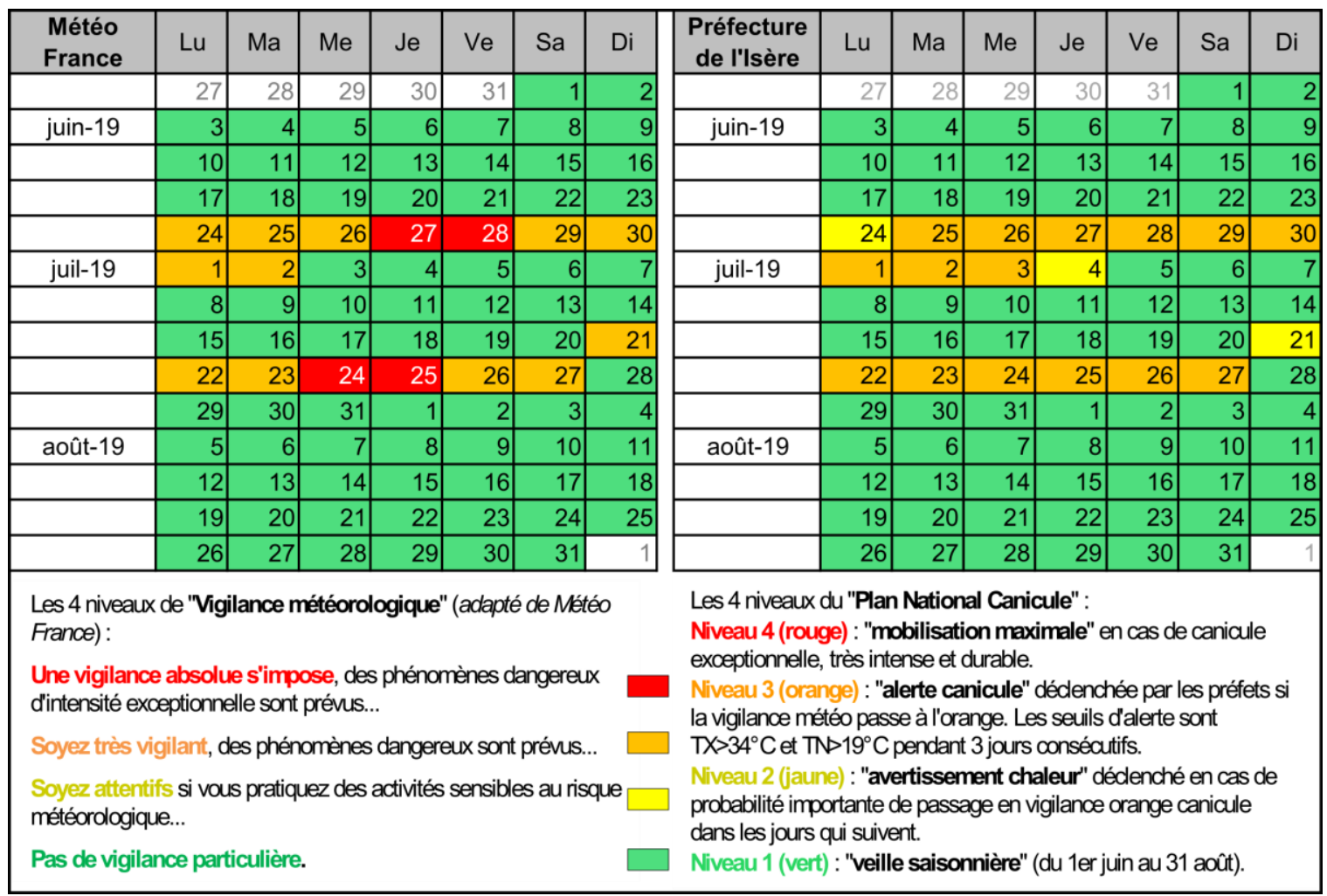

Météo France a ainsi placé 12 jours de l'été en vigilance orange et 4 en rouge (tableau 4). L'activation du niveau 3 (orange) du Plan Canicule est donc fortement tributaire des alertes météo et n'est réalisée que si le risque sanitaire est important. Cette activation a été plus fréquente en 2019 que les années précédentes, en Isère comme sur le territoire national, compte-tenu de l'intensité exceptionnelle des chaleurs enregistrées; aux alertes canicule et PNC s'ajoutent des actions préventives de l'agglomération dont certaines ont été déclenchées dans l'urgence de 2019 lorsque le niveau d'alerte 3 canicule a été annoncé par le Préfet de l'Isère. L'intensité et la durée de la vague de chaleur ont donc eu un effet sur la réorganisation des services de la collectivité, non seulement pendant 
l'événement, mais également à plus long terme, en vue de capitaliser sur l'expérience acquise et préparer l'été suivant. Ces ajustements ont par exemple permis de constituer une cellule de crise et d'améliorer la procédure de transmission de l'alerte préfectorale à l'ensemble de la chaîne managériale par le service sécurité civile de la Ville. Mais à ce jour, elles n'ont toujours pas été calculées à l'échelle des gradations du risque en fonction des échelles infra-urbaines (par exemple en tenant compte des Local Climate Zone - LCZ).

\section{Conclusion}

L'analyse rétrospective de l'épisode chaud extrême enregistré durant l'été 2019 à Grenoble apporte plusieurs éléments de prospective pour anticiper le risque climatique urbain à court et à moyen termes. Les résultats semblent indiquer que cette vague de chaleur sera potentiellement un événement chaud 'commun' d'ici 2050 et encore plus au-delà. Deux grandes prochaines étapes sont d'une part, de renforcer les connaissances métrologiques à l'échelle de la mosaïque urbaine, et d'autre part, de mieux cartographier les contraintes imposées par l'îlot de chaleur urbain. Cela doit passer par d'autres mesures et stations que celles existant à ce jour, et même si l'apport de la station CEA-Radôme depuis l'automne 2019 est un atout ; or ce référentiel urbain demeure toujours insuffisant car sous-estime les aléas thermiques, ce dont les responsables de Météo France ont bien conscience (Taboulot, 2019).

Très engagée et impliquée avec la communauté scientifique, la municipalité poursuit ses actions opérationnelles et d'adaptation aux aléas caniculaires, avec par exemple l'ouverture nocturne de trois parcs urbains (habituellement fermés la nuit), la gratuité des musées, mais aussi l'édition d'une carte de recensement des points de fraîcheur à Grenoble. Des actions d'urbanisme stratégique à moyen terme visent la végétalisation de l'espace public et privé (façades et toitures), la désimperméabilisation des sols ou une présence renforcée de l'eau dans la ville, en tenant compte du confort thermique estival dans la rénovation du patrimoine municipal et dans les nouvelles constructions (en lien avec le schéma directeur de l'immobilier). Ceci est réalisé non seulement à l'échelle de l'agglomération urbaine mais également en identifiant les points chauds où des actions prioritaires doivent être effectuées. Plusieurs autres actions en préparation proviennent du retour d'expérience des extrêmes subis en 2019, comme certaines solutions techniques apportées aux bâtiments pour limiter l'inconfort thermique (jeux d'eau, partage des pièces les plus fraîches, etc.). A court terme, les programmations fédérées de la Ville et celles de Grenoble Alpes Métropole (communauté d'agglomération) aideront à mieux lutter contre les extrêmes météo-climatiques futurs.

Remerciements : Les auteurs remercient les organismes ayant fourni certaines données et informations : 1) l'Institut Max von Laue - Paul Langevin (ILL) du CEA et en particulier Giovanna Cicognani, Frédéric Fouladoux et Jean-Philippe Pierre ; 2) ATMO-AURA ; 3) Météo-France via la Publithèque et le portail Drias ; 4) Brice Boudevillain de l'OSUG-IGE ; 5) la DDT de l'Isère et 6) la Préfecture de l'Isère.

\section{Références}

Alonso L. et Renard F., 2020. A comparative study of the physiological and socio-economic vulnerabilities to heatwaves of the population of the Metropolis of Lyon (France) in a climate change context. Int. J. Environ. Res. Public Health, 17, 1004; doi:10.3390/ijerph17031004.

Bador M., 2016. Les changements d'extrêmes de température en Europe : records, canicules intenses et influence anthropique. Thèse de doctorat, Université Paul Sabatier, Toulouse, 255 pages.

Baklanov A., Cardenas B., Lee T., Leroyer S., Masson V., Molina L. T., Muller T., Ren T., Vogel F. R., Voogt J. A., 2020. Integrated urban services: Experience from four cities on different continents. Urban Climate, 32, 100610.

Barlow J. F., 2014. Progress in observing and modelling the urban boundary layer. Urban Climate, 10, 216-240.

Bigot S., Duché S., Madelin M. et Rome S., 2017. Etude du climat urbain : pour une mise à disposition de nouveaux services climatiques. BSGLg [En ligne], 68, http://popups.uliege.be/0770-7576/index.php?id=4505.

BSP (Bulletin de Santé Publique), 2019. Canicule et santé Été 2019. Santé Publique France, 9 pages.

Chao R., Yan-Yung N. E., Lutz K., 2010. Urban climatic map studies: a review. International Journal of Climatology, 31 (15), 2213-2233, doi: 10.1002/joc.2237.

Copernicus, 2020. European state of the climate 2019 - 
Summary. European Commission and Copernicus - ECMWF, 13 pages.

Daniel M., 2017. Villes, climat urbain et climat régional sur la France : étude par une approche de modélisation climatique couplée. Thèse de doctorat, Université Toulouse 3 Paul Sabatier, 193 pages.

Foissard X., Dubreuil V., Quénol H., 2019. Defining scales of the land use effect to map the urban heat island in a mid-size European city: Rennes (France). Urban Climate, 29, 100490.

Kalnay E., Kanamitsu M., Kistler R. et al., 1996. The NCEP/NCAR 40-year reanalysis project. Bull. Amer. Meteor. Soc., 77, 437-470.

Largeron Y., 2010. Dynamique de la Couche Limite Atmosphérique stable en relief complexe. Application aux épisodes de pollution particulaire des vallées alpines. Thèse de doctorat, Université Joseph Fourier, Grenoble, 307 pages.

Musco F., Appiotti F., Bianchi I., Dalla Fontana M., Gissi E., Lucertini G., Magni F. and Maragno D., 2016. Planning and climate change: concepts, approaches, design. In: Counteracting urban heat island effects in a global climate change scenario, Francesco Musco Ed., Venice, Italy, Springer Open, 35-44.

Oke T. R. 1982. The energetic basis of the urban heat island. Quarterly Journal of the Royal Meteorological Society, 108(455), 1-24.

Ouzeau G., Soubeyroux J.-M., Schneider M., Vautard R., Planton S., 2016. Heat waves analysis over France in present and future climate: Application of a new method on the EURO-CORDEX ensemble. Climate Services, 4, 1-12.

PNC, 2017. Plan National Canicule. Ministère des Solidarités et de la Santé, France, Paris, 51 pages.

Préfecture de l'Isère, 2016. Plan départemental de gestion d'une canicule en Isère, Juin 2016.

Rome S., Bigot S., Foissard X., Fouvet A.-C., Madelin M. et
Duché S. (2020). L'été 2019 à Grenoble (France) : analyse d'un extrême de chaleur et mesures d'adaptations mises en place par la collectivité. In « Changement climatique et Territoires » (ed. Bonnardot V. et Quénol H.), Actes du colloque de l'AIC, 33, Rennes, France, 601-610.

Santé Publique France (SPF), 2019. Canicule et santé. Bulletin de santé publique. Été 2019.

Singh R., Arrighi J., Jjemba E., Strachan K., Spires M., Kadihasanoglu A., 2019. Heatwave Guide for Cities. Red Cross Red Crescent Climate Centre, 96 pages.

Soubeyroux J.-M., Ouzeau G., Schneider M., Cabanes O. \& Kounkou-Arnaud R., 2016. Les vagues de chaleur en France : analyse de l'été 2015 et évolutions attendues en climat futur. La Météorologie, 94, 45-51. DOI : https://doi.org/10.4267/2042/60704

Suarez-Gutierrez L., Müller W. A., Li C., Marotzke J., 2020. Dynamical and thermodynamical drivers of variability in European summer heat extremes. Climate Dynamics, DOI: $10.1007 / \mathrm{s} 00382-020-05233-2$.

Taboulot S., 2020. Grenoble a (enfin) une station météo... Le Dauphiné Libéré, 04 oct. 2019 : https://www.ledauphine.com/iseresud/2019/10/03/grenoble-a-(enfin)-une-station-meteo

Theeuwes N. E., Ronda R. J., Harman I. N., Christen A., Grimmond C. S. B., 2019. Parametrizing HorizontallyAveraged Wind and Temperature Profiles in the Urban Roughness Sublayer. Boundary-Layer Meteorology, 173, 321348 .

Vautard R., Boucher O., van Oldenborgh G. J., Otto F., Haustein K., Vogel M. M., Seneviratne S. I., Soubeyroux J.M., Schneider M., Drouin A., Ribes A., Kreienkamp F., Stott P., van Aalst M., 2019. Human contribution to the recordbreaking July 2019 heat wave in Western Europe. Rapport scientifique, IPSL, Paris, 32 pages.

Ville de Grenoble, 2019. Plan Municipal Canicule (PNC) 2020-2022. 18 octobre 2019.

Citation de l'article : Rome et al., 2020. Les deux épisodes caniculaires de l'été 2019 à Grenoble : constat et perspective pour une gestion des extrêmes thermiques futurs. Climatologie, 17, 12. 